\title{
Predictors of outcome after alcohol septal ablation in patients with hypertrophic obstructive cardiomyopathy. Special interest for the septal coronary anatomy
}

\author{
R. C. Steggerda • J. C. Balt • K. Damman • \\ M. P. van den Berg • J. M. ten Berg
}

Published online: 24 July 2013

(C) The Author(s) 2013. This article is published with open access at Springerlink.com

\begin{abstract}
Background Alcohol septal ablation (ASA) provides symptomatic relief in most but not all patients with hypertrophic obstructive cardiomyopathy (HOCM). Therefore we investigated predictors of outcome after ASA.

Methods Clinical, echocardiographic, angiographic and procedural characteristics were analysed in 113 consecutive patients. Successful ASA was defined as NYHA $\leq 2$ with improvement of at least 1 class combined with a resting gradient $<30 \mathrm{mmHg}$ and provoked gradient $<50 \mathrm{mmHg}$ at 4-month follow-up.

Results In 37 patients ASA was not successful. In multivariate analysis, baseline gradient (OR $1.06(1.01-1.11)$ per $5 \mathrm{mmHg}, p=0.024$ ) and distance to the ablated septal branch (OR $1.09(1.03-1.16)$ per $\mathrm{mm}, p=0.004)$ were predictors of unsuccessful outcome. The combined presence of a nonablated septal branch and a distance $\geq 19 \mathrm{~mm}$ to the ablated branch was a predictor of unsuccessful outcome (OR 5.88 $(2.06-16.7), p<0.001)$.

Conclusions Baseline gradient and a greater distance from the origin of the left anterior descending artery to the ablated septal branch combined with a non-ablated proximal septal branch are associated with an unsuccessful outcome after ASA.
\end{abstract}

R. C. Steggerda $(\bowtie)$

Department of Cardiology, Martini Hospital, Van Swietenplein 1, 9728 NT Groningen, the Netherlands

e-mail: r.c.steggerda@mzh.nl

J. C. Balt $\cdot$ J. M. ten Berg

Department of Cardiology, St. Antonius Hospital, Nieuwegein, the Netherlands

K. Damman • M. P. van den Berg

Department of Cardiology, University of Groningen, University

Medical Center Groningen, Groningen, the Netherlands
Keywords Hypertrophic obstructive cardiomyopathy · Left ventricular outflow tract · Alcohol septal ablation · Outcome . Septal anatomy

\section{Introduction}

In patients with symptomatic hypertrophic obstructive cardiomyopathy (HOCM), percutaneous alcohol septal ablation (ASA) is an accepted alternative to surgical septal myectomy ('myectomy') [1-3]. Although no randomised controlled trials have been performed, observational meta-analyses for ASA versus myectomy show excellent long-term survival rates for both procedures [4-7]. However, reduction of the gradient in the left ventricular outflow tract (LVOT) appears to be slightly less for patients who undergo ASA [4]; repeat interventions are performed more often after ASA [8] and in a single-centre study cardiac death occurred more often after ASA compared with myectomy [9]. With myectomy, the basal part of the septum, responsible for the obstruction of the LVOT, is usually resected completely. In contrast, after ASA the most basal part of the septum may in some cases be spared causing residual obstruction [10]. In this study, we studied the predictors of outcome after ASA with special interest for the septal coronary anatomy.

\section{Methods \\ Patients}

We studied patients who were treated for HOCM at the St. Antonius Hospital in Nieuwegein, the Netherlands. For patients to be selected for septum reduction (either ASA or myectomy) they had to have severe symptoms (New York 
Heart Association functional class $\geq 3$ ) despite trial of optimal medical therapy in combination with a resting gradient in the left ventricular outflow tract $($ LVOT) $\geq 30 \mathrm{mmHg}$ and/or a provocable gradient $\geq 50 \mathrm{mmHg}$. All patients were required to have left ventricular asymmetrical septal hypertrophy, with a minimal septum diameter $\geq 15 \mathrm{~mm}$. Patients with concomitant (sub)valvular disease or other conditions that warranted surgery were accepted to undergo surgical myectomy. Patients who were eligible for both options were informed about the known risks and benefits of both ASA and surgical myectomy and were offered the choice between these procedures. Routine clinical and echocardiographic assessments were performed in all patients. We performed a retrospective analysis of all the clinical data along with analysis of the echocardiographic images and coronary angiograms. Civil registries were used to determine survival in January 2011 . The study conformed to principles defined in the Helsinki Declaration.

\section{Definitions}

Successful symptomatic relief was defined as a New York Heart Association (NYHA) functional class $\leq 2$ with a lowering of at least one NYHA class at follow-up. The combination of both successful symptomatic relief with a resting gradient $<30 \mathrm{mmHg}$ and a provoked gradient $<50 \mathrm{mmHg}$ during echocardiographic assessment at 4-month follow-up was used as a strict definition for successful outcome.

\section{Alcohol septal ablation}

ASA was performed as described in detail previously [11]. In short, with the aid of a flexible coronary guide wire, a coronary balloon was placed in the most proximal septal branch. Myocardial contrast echocardiography was used for further guidance. When the region of contrast in the septum was satisfactory and adjacent to the area of septal contact of the anterior mitral valve leaflet, 0.5 to $3 \mathrm{ml}$ of concentrated ethanol was slowly injected through the inflated balloon catheter. The balloon was left inflated for $10 \mathrm{~min}$ to prevent retrograde spill of ethanol. Invasive gradients in the LVOT were measured continuously during the procedure using a 6 French pigtail catheter inserted in the left ventricle. For testing of a provocable gradient, the Valsalva manoeuvre and extrasystolic beats were used. When the gradient in the LVOT remained $\geq 30 \mathrm{mmHg}$ after the first ablation (either at rest or after provocation), the procedure was repeated in a second septal branch. During the procedure, all patients received a temporary transvenous pacemaker. If an AV block remained more than $48 \mathrm{~h}$ after the procedure, a definitive pacemaker was implanted.
Echocardiography

All echocardiograms were performed with Hewlett-Packard Sonos 5500 and Philips IE33 ultrasound imaging systems and were stored as digital images in a database. Philips' Excelera software program was used for all measurements, which were performed by a single observer (RCS) who was blinded for the purpose of the study. Measurement of the peak LVOT gradient both at rest and after provocation using the Valsava manoeuvre was obtained in the apical 3 or 5 chamber view. The degree of mitral insufficiency (MI) was graded from 0 to 4 (grade $0=$ no MI, grade $1=$ mild, grades 2 and $3=$ moderate, grade $4=$ severe) [12]. The degree of systolic anterior motion (SAM) was graded from 0 to 4 (grade $0=$ no SAM, grade $1=\mathrm{SAM}$ with distance from septum to AMVL $>10 \mathrm{~mm}$, grade $2=\mathrm{SAM}$ with distance from septum to $\mathrm{AMVL}<10 \mathrm{~mm}$, grade $3=\mathrm{AMVL}$ makes brief septal contact, grade $4=$ septal contact $>30 \%$ during systole) [13]. The minimal LVOT diameter was measured as the narrowest part in the LVOT between the septum and the basis of the anterior mitral valve leaflet (AMVL) during systole. At 4 months of follow-up after ASA, the baseline measurements were repeated and in addition, in the parasternal long-axis view, septal thinning due to the infarction was evaluated at end-diastole. When septal thinning was found from the beginning of the septum, the infarction was considered to be without sparing of the basal septum. Otherwise, the infarction was considered to have spared the basal septum. Akinesia of the basal septum was rated as either present or absent.

\section{Coronary angiography}

Coronary angiography was usually performed shortly before the procedure. For the purpose of the present study, the coronary angiograms of all patients were retrospectively analysed for the presence of non-ablated proximal septal branches. All branches that clearly perfused the septum and were proximal to the first ablated septal branch were considered non-ablated septal branches. Furthermore, using Philips suite QCA with the catheter diameter for calibration, we measured the distance from the origin of the LAD to the origin of the ablated septal branch. We also measured the diameters of the origin of both the non-ablated proximal septal branch and the ablated septal branches.

\section{Statistical analysis}

Data are given as the mean and standard deviation when normally distributed, as the median with interquartile range for skewed distributions, and as frequencies and percentages for categorical variables. The Student's $t$-test was used for continuous variables and the chi-square test was used to 
compare categorical variables. Pearson's test was used for calculating correlations. Logistic regression was carried out to identify univariate predictors of unsuccessful outcome. Stepwise backward logistic regression analysis was carried out in a multivariate analysis. Variables were considered in the multivariate models when a $\mathrm{P}$-value of $<0.1$ was obtained in the univariate analysis. In secondary analysis, interaction analysis was carried out to establish significant interactions between the variables of septal coronary anatomy and unsuccessful outcome. All reported probability values are twotailed, a $P$-value $<0.05$ was considered statistically significant and a $\mathrm{P}$-value $<0.1$ was considered significant for interaction analysis. Statistical analyses were performed using STATA, College Station, Texas, version 10.0.

\section{Results}

A dataset of 113 patients who underwent ASA in our institution from January 2000 to December 2009 was completed. At 4-month follow-up a successful symptomatic result was found in $90 \%$ of all patients. The total group showed reduction of the echocardiographic gradient both at rest and after provocation from $50 \mathrm{mmHg} \pm 43$ and $113 \mathrm{mmHg} \pm 61$, respectively, before the procedure to $22 \mathrm{mmHg} \pm 26$ and $38 \mathrm{mmHg} \pm 40$, measured 4 months after ASA $(p<0.001)$. According to the definition of the present study, ASA was successful in 76 patients (successful group) and unsuccessful in 37 patients (unsuccessful group). The baseline characteristics of the patients in the two groups did not differ significantly (Table 1). At 4 months after the procedure, the NYHA functional class had improved, resulting in a post-procedural NYHA functional class of $1.0 \pm 0.6$ in the successful group versus $1.9 \pm 1.0$ in the unsuccessful group $(p<0.001)$.

In terms of peri-procedural complications ventricular fibrillation occurred once and was treated with defibrillation, tamponade occurred once and was treated with pericardiocentesis and dissection in the LAD occurred once and was treated with a stent implantation. A definitive pacemaker was inserted in five patients. During a follow-up of $5.3 \pm 2.5$ years there were 4 deaths ( 3 non-cardiac, 1 cardiac), amounting an annual all-cause mortality of $0.7 \%$.

\section{Echocardiographic findings}

Echocardiographic findings at baseline and at 4-month follow-up comparing both groups are shown in Table 2 . Before ASA, the resting gradient in the LVOT was higher in the unsuccessful group than in the successful group, but the other characteristics were comparable. Inherent to the definition of successful outcome, after ASA the LVOT gradient in the unsuccessful group was higher than in the successful group. Furthermore, in the unsuccessful group, infarctions with sparing of the basal septum were more common than in the successful group. Of note, infarctions without sparing of the basal septum were associated with a larger minimal

Table 1 Baseline characteristics

Data are presented as mean \pm standard deviation or as percentage (number)

$A S A$ alcohol septal ablation, $C C S$ Canadian Cardiovascular Society, CK creatine kinase, NYHA New York Heart Association

\begin{tabular}{lccc}
\hline Variables & \multicolumn{2}{l}{ Outcome after ASA } & \multirow{2}{*}{$P$-value } \\
\cline { 2 - 3 } & Successful $(n=76)$ & Unsuccessful $(n=37)$ & \\
\hline Age (years) & $56 \pm 17$ & $57 \pm 14$ & 0.93 \\
Male sex (\% (n)) & $55(41)$ & $44(17)$ & 0.32 \\
NYHA functional class & $2.9 \pm 0.4$ & $2.8 \pm 0.7$ & 0.78 \\
Angina functional class & $1.9 \pm 0.4$ & $2.0 \pm 0.4$ & 0.33 \\
Syncope (\% (n)) & $23(17)$ & $18(7)$ & 0.71 \\
3-blocker or Ca-blocker (\% (n)) & $83(63)$ & $78(29)$ & 0.52 \\
Previous pacemaker (\% (n)) & $5(4)$ & $0(0)$ & 0.36 \\
Max CK (U/l) & $1294 \pm 461$ & $1431 \pm 793$ & 0.34 \\
Max CK Mb (U/l) & $201 \pm 124$ & $167 \pm 59$ & 0.051 \\
Hospital stay, days & $5.6 \pm 4$ & $6.3 \pm 5$ & 0.49 \\
Angiographic findings & & & \\
- Non-ablated branch present (\% (n)) & $26(19)$ & $50(19)$ & 0.01 \\
- Distance to ablated branch (mm) & $16 \pm 7$ & $20 \pm 8$ & 0.006 \\
- Diameter ablated branch (mm) & $1.7 \pm 0.4$ & $1.6 \pm 0.5$ & 0.25 \\
- Diameter non-ablated branch (mm) & $1.0 \pm 0.3$ & $1.1 \pm 0.4$ & 0.32 \\
- Amount of alcohol (ml) & $2.4 \pm 0.7$ & $2.3 \pm 0.5$ & 0.55 \\
- Number of ablated branches & $1.2 \pm 0.4$ & $1.2 \pm 0.4$ & 0.99 \\
- Invasive gradient pre ASA (mmHg) & $83 \pm 32$ & $95 \pm 35$ & 0.08 \\
\hline
\end{tabular}


Table 2 Echocardiographic findings at baseline and 4 months follow up
Data are presented as mean \pm standard deviation or as percentage (number)

LVOT left ventricular outflow tract, $M I$ Mitral insufficiency, $S A M$ systolic anterior motion

\begin{tabular}{|c|c|c|c|}
\hline \multirow{2}{*}{$\begin{array}{l}\text { Variables } \\
\text { Baseline }\end{array}$} & \multicolumn{2}{|l|}{ Outcome after ASA } & \multirow[t]{2}{*}{$P$ value } \\
\hline & Successful $(n=76)$ & Unsuccessful $(n=37)$ & \\
\hline Septal thickness (mm) & $22 \pm 5$ & $22 \pm 5$ & 0.95 \\
\hline Posterior wall thickness (mm) & $15 \pm 3$ & $16 \pm 3$ & 0.71 \\
\hline End-diastolic volume (ml) & $77 \pm 25$ & $74 \pm 26$ & 0.63 \\
\hline End-systolic volume (ml) & $18 \pm 11$ & $16 \pm 7$ & 0.20 \\
\hline Ejection fraction (\%) & $76 \pm ?$ & $78 \pm ?$ & 0.41 \\
\hline LVOT $\min (\mathrm{mm})$ & $14 \pm 5$ & $13 \pm 5$ & 0.66 \\
\hline Degree of MI & $1.5 \pm 1.0$ & $1.7 \pm 1.1$ & 0.50 \\
\hline Degree of SAM & $2.9 \pm 0.6$ & $3.0 \pm 0.6$ & 0.14 \\
\hline Resting gradient (mmHg) & $43 \pm 40$ & $63 \pm 46$ & 0.026 \\
\hline Provoked gradient (mmHg) & $107 \pm 53$ & $126 \pm 67$ & 0.15 \\
\hline \multicolumn{4}{|l|}{ At 4-month follow-up } \\
\hline Septal thickness (mm) & $20 \pm 4$ & $20 \pm 4$ & 0.43 \\
\hline Posterior wall thickness (mm) & $14 \pm 3$ & $14 \pm 3$ & 0.62 \\
\hline End-diastolic volume (ml) & $81 \pm 27$ & $74 \pm 24$ & 0.14 \\
\hline End-systolic volume (ml) & $23 \pm 15$ & $18 \pm 9$ & 0.053 \\
\hline Ejection fraction $(\%)$ & $74 \pm 10$ & $77 \pm 10$ & 0.16 \\
\hline LVOT $\min (\mathrm{mm})$ & $19 \pm 4$ & $15 \pm 5$ & $<0.001$ \\
\hline LVOT min, increase & $5.6 \pm 4$ & $1.7 \pm 3$ & $<0.001$ \\
\hline Degree of MI & $0.5 \pm 0.7$ & $1.0 \pm 1.0$ & 0.020 \\
\hline Degree of SAM & $1.0 \pm 0.9$ & $2.2 \pm 1.1$ & $<0.001$ \\
\hline Akinetic basal septum (\% (n)) & $72(55)$ & $19(7)$ & $<0.001$ \\
\hline Without sparing basal septum (\% (n)) & $58(45)$ & $17(6)$ & $<0.001$ \\
\hline Resting gradient $(\mathrm{mmHg})$ & $12 \pm 7$ & $41 \pm 38$ & $<0.001$ \\
\hline Provoked gradient (mmHg) & $17 \pm 10$ & $91 \pm 53$ & $<0.001$ \\
\hline Decrease provoked gradient $(\mathrm{mmHg})$ & $91 \pm 53$ & $35 \pm 60$ & $<0.001$ \\
\hline
\end{tabular}

LVOT $(20 \pm 4$ vs $15 \pm 4, p<0.001)$ and a lower grade of SAM $(0.7 \pm 0.6$ vs $2.0 \pm 1.1, p<0.001)$ compared with infarctions with sparing.

\section{Angiographic findings}

The angiographic findings are shown in Table 1. In the unsuccessful group, there were more patients with a nonablated proximal septal branch and the distance from the origin of the LAD to the ablated branch was greater. In both groups the diameter of the first ablated branch was larger than the diameter of the non-ablated proximal septal branch ( $p<0.01$ for both groups).

\section{Predictors of unsuccessful outcome}

Of all the clinical and echocardiographic characteristics at baseline, only baseline resting gradient at echocardiography predicted outcome. Of the angiographic characteristics, both the presence of a non-ablated proximal septal branch and the distance to the ablated septal branch were predictors of outcome (Table 3 ). After multivariate analysis, only the distance to the ablated branch and baseline resting gradient were found to be predictive for outcome after ASA (Table 3). Secondary analysis revealed a significant interaction between the distance to the ablated branch and the presence of a nonablated proximal septal branch when the distance was dichotomised based on the $19 \mathrm{~mm}$ optimal cut-off point (using highest sensitivity and specificity) (P-value for interaction 0.025). The presence of a non-ablated proximal septal branch and a distance $\geq 19 \mathrm{~mm}$ to the ablated branch predicted an unsuccessful outcome (OR 5.88 (2.06-16.74), $P<0.001$ ), Table 4) Of note, in this subgroup of patients $(n=27 / 113$, $24 \%$ ), sparing of the basal septum was more common (55\% versus $15 \%, p<0.001)$ compared with the other patients.

\section{Discussion}

Symptomatic relief was found in $90 \%$ of patients, comparable with previous results $[1,14]$, but success of ASA in terms of reduction of the gradient was somewhat less common. We found that a greater distance from the origin of the 
Table 3 Univariate and multivariate predictors of unsuccessful outcome

\begin{tabular}{|c|c|c|c|c|}
\hline \multirow[t]{2}{*}{ Variable } & \multicolumn{2}{|l|}{ Univariate } & \multicolumn{2}{|l|}{ Multivariate } \\
\hline & OR $(95 \% \mathrm{CI})$ & $P$-value & OR $(95 \% \mathrm{CI})$ & $P$-value \\
\hline Age & $1.00(0.97-1.03)$ & 0.87 & & \\
\hline Sex (males) & $1.50(0.68-3.32)$ & 0.32 & & \\
\hline Baseline resting gradient (per $5 \mathrm{mmHg}$ ) & $1.06(1.01-1.11)$ & 0.024 & $1.06(1.01-1.11)$ & 0.024 \\
\hline Distance to ablated branch (per mm) & $1.08(1.03-1.55)$ & 0.005 & $1.09(1.03-1.16)$ & 0.004 \\
\hline Non-ablated branch present & $2.85(1.24-6.53)$ & 0.013 & & \\
\hline
\end{tabular}

$O R$ odds ratio

LAD to the ablated septal branch, especially in the presence of a non-ablated proximal septal branch, was associated with an unsuccessful outcome 4 months after ASA, independent of the severity of the baseline resting gradient. In terms of safety, mortality rate was low. Complication rates in our study were low including the number of pacemaker implantations, probably due to the low amounts of alcohol we used based on careful assessment with myocardial contrast echocardiography.

\section{Unfavourable coronary anatomy}

As previously demonstrated using cardiac MRI, patients exhibit a more variable location of septal thinning after ASA compared with surgical myectomy [10]. The access to the perfusion bed of the most basal part of the septum may for the interventional cardiologist be limited or difficult due to the variability of the septal anatomy [15-17]. Even though ASA was only performed after contrast was seen in the region of interest using myocardial contrast echocardiography, we also found a considerable variation of the location of the infarction in our study. Moreover, an unfavourable anatomy (combined presence of a non-ablated proximal septal branch and a distance $\geq 19 \mathrm{~mm}$ to the ablated branch) was associated with an unsuccessful outcome. In these patients more frequent sparing of the most basal septum was found accompanied by a smaller minimal LVOT diameter and a higher grade of SAM. This may explain a lower success rate of ASA in patients with an unfavourable anatomy. For these patients, surgical myectomy might thus be a preferred method instead of ASA. The effect of an unfavourable anatomy on long-term survival and clinical outcome warrants further study.

\section{Limitations}

This was a retrospective single-centre study. Measurements were performed on the available angiographic images but we cannot rule out that more targeted imaging might have yielded more anatomically correct values. We used a strict definition for success consisting of a combined symptomatic result with the same haemodynamic criteria for invasive treatment mentioned in current guidelines [18]; however, there is no clear consensus for definition of success after ablation. The choice of the endpoint at 4 months was a practical one, simply based on the first contact at the outpatient clinic. It cannot be excluded that further improvements in symptomatic status and LVOT gradients occurred beyond 4 months after ASA.

Table 4 Multivariate predictors of successful outcome taking into account the interaction between the distance to the ablated branch and the presence of a non-ablated branch

\begin{tabular}{|c|c|c|c|c|c|}
\hline \multirow[t]{2}{*}{ Variable } & \multirow[t]{2}{*}{$\mathrm{N}$} & \multicolumn{2}{|l|}{ Univariate } & \multicolumn{2}{|l|}{ Mulitvariate } \\
\hline & & OR $(95 \% \mathrm{CI})$ & $P$-value & OR $(95 \% \mathrm{CI})$ & $P$-value \\
\hline Baseline resting gradient (per $5 \mathrm{mmHg}$ ) & & $1.06(1.01-1.11)$ & 0.024 & $1.05(1.00-1.10)$ & 0.061 \\
\hline \multicolumn{6}{|l|}{ Distance vs non-ablated branch } \\
\hline - Distance $<19 \mathrm{~mm}$, non-ablated branch not present & 54 & 1.00 & - & 1.00 & - \\
\hline - Distance $\geq 19 \mathrm{~mm}$, non-ablated branch not present & 21 & $0.99(0.30-3.21)$ & 0.98 & $1.14(0.33-3.88)$ & 0.83 \\
\hline - Distance $<19 \mathrm{~mm}$, non-ablated branch present & 11 & $0.32(0.36-2.70)$ & 0.29 & $0.40(0.05-3.52)$ & 0.41 \\
\hline - Distance $\geq 19 \mathrm{~mm}$, non-ablated branch present & 27 & $5.36(1.97-14.57)$ & 0.001 & $5.88(2.06-16.74)$ & 0.001 \\
\hline
\end{tabular}

$O R$ odds ratio

Distance: the measured distance from the origin of the LAD to the origin of the ablated septal branch 


\section{Conclusions}

Baseline gradient and the combined presence of a nonablated proximal of septal branch with a greater distance to the ablated septal branch was found to be associated with an unsuccessful outcome after ASA. In practical terms this implies that ASA should probably not be performed when septal anatomy is unfavourable.

Acknowledgments We thank Jackie Senior for editing the manuscript.

\section{Funding None.}

Conflict of interests None declared.

Open Access This article is distributed under the terms of the Creative Commons Attribution License which permits any use, distribution, and reproduction in any medium, provided the original author(s) and the source are credited.

\section{References}

1. Fifer MA, Sigwart U. Hypertrophic obstructive cardiomyopathy: alcohol septal ablation. Eur Heart J. 2011;32:1059-64.

2. Maron BJ, Maron MS, Wigle ED, et al. The 50-year history, controversy and clinical implications of left ventricular outflow tract obstruction in hypertrophic cardiomyopathy: from idiopathic hypertrophic subaortic stenosis to hypertrophic cardiomyopathy. J Am Coll Cardiol. 2009;54:191-200.

3. ten Berg J, Steggerda RC, Siebelink HM. Myocardial disease: the patient with hypertrophic cardiomyopathy. Heart. 2010;96:1764-72.

4. Alam M, Dokainish H, Lakkis NM. Hypertrophic obstructive cardiomyopathy-alcohol septal ablation vs myectomy: a metaanalysis. Eur Heart J. 2009;30:1080-7.

5. Leonardi RA, Kransdorf EP, Simel DL, et al. Meta-analysis of septal reduction therapies for obstructive hypertrophic cardiomyopathy. Comparative rates of overall mortality and sudden cardiac death after treatment. Circ Cardiovasc Interv. 2010;3:1-7.
6. Agarwal S, Tuzcu EM, Desai MY, et al. Updated meta-analysis of septal alcohol ablation versus myectomy for hypertrophic cardiomyopathy. J Am Coll Cardiol. 2010;55:823-34.

7. Ball W, Ivanov J, Rakowski H, et al. Long-term survival in patients with resting obstructive hypertrophic cardiomyopathy comparison of conservative versus invasive treatment. J Am Coll Cardiol. 2011;58:2313-21.

8. Alam M, Dokainish H, Lakkis N. Alcohol septal ablation for hypertrophic obstructive cardiomyopathy: a systematic review of published studies. J Interv Cardiol. 2006;19:319-27.

9. ten Cate FJ, Soliman O, Michels M, et al. Long-term outcome of alcohol septal ablation in patients with obstructive hypertrophic cardiomyopathy: a word of caution. Circ Heart Fail. 2010;3(3):362-9.

10. Valeti US, Nishimura RA, Holmes DR, et al. Comparison of surgical septal myectomy and alcohol septal ablation with cardiac magnetic resonance imaging in patients with hypertrophic obstructive cardiomyopathy. J Am Coll Cardiol. 2007;49:350-7.

11. Van der Lee C, Scholzel B, ten Berg JM, et al. Usefulness of clinical, echocardiographic, and procedural characteristics to predict outcome after percutaneous transluminal septal myocardial ablation. Am J Cardiol. 2008;101:1315-20.

12. Destefano FR. Determination of mitral regurgitation severity. In: Armstrong WF, Ryan T, editors. Feigenbaum's echocardiography. 7th ed. Philadelphia: Lippincott Williams and Wilkins; 2009. p. $320-5$.

13. Pollick C, Rakowski H, Wigle ED. Muscular subaortic stenosis: the quantative relationship between systolic aortic motion and pressure gradient. Circulation. 1984;69:43-9.

14. Nagueh SF, Groves BM, Schwartz L, et al. Alcohol septal ablation for the treatment of hypertrophic obstructive cardiomyopathy. J Am Coll Cardiol. 2011;58:2322-8.

15. Singh M, Edwards WD, Holmes DR, et al. Anatomy of the first septal perforating artery: a study with implications for ablation therapy for hypertrophic cardiomyopathy. Mayo Clin Proc. 2001;76: 799-802.

16. Brinkjikji W, Harris SR, Froemming AT, et al. Descriptive anatomy of the dominant septal perforators using dual source coronary CT angiography. Clin Anat. 2010;23:70-8.

17. Steggerda RC, Balt JC, ten Berg JM. Optimal gradient reduction after alcohol septal ablation: a case report with anatomical and practical determinants. Neth Heart J. 2011;19(6):301-3.

18. Gersh BJ, Maron BJ, Bonow RO, et al. 2011 ACCF/AHA guideline for the diagnosis and treatment of hypertrophic cardiomyopathy. $\mathrm{J}$ Am Coll Cardiol. 2011;58:e212-60. 